Editorial: Hospital Metropolitano

ISSN (impreso) 1390-2989 - ISSN (electrónico) 2737-6303

Edición: Vol. 28 No 3 (2020) Julio - Septiembre

DOI: https://doi.org/10.47464/MetroCiencia/vol28/3/2020/39-48

URL: https://revistametrociencia.com.ec/index.php/revista/article/view/85

Pág: $39-48$

\title{
Efectividad de esquemas de terapia respiratoria respecto a oxigenoterapia y estancia en lactantes con bronquiolitis
}

\section{Effectiveness of respiratory therapy schemes with respect to oxygen therapy and stay in infants with bronchiolitis}

\author{
Dayana Navarro' ${ }^{1 D}$; Daniela Briceño' \\ Médica Pediatra, Pontificia Universidad Católica del Ecuador, Quito-Ecuador
}

Recibido: 20/06/2020 Aceptado: 10/07/2019 Públicado: 01/09/2020

\begin{abstract}
RESUMEN
Introducción: La bronquiolitis está entre las principales causas de morbilidad en menores de 24 meses. Objetivo: Determinar la efectividad de los esquemas de terapia respiratoria respecto al tiempo de estancia hospitalaria y requerimiento de oxígeno en menores de 2 años, ingresados con bronquiolitis en el Hospital Metropolitano desde enero del 2014 a diciembre del 2019. Método: Estudio analítico retrospectivo, 546 pacientes con bronquiolitis y oxigenoterapia. El análisis univariado de variables cuantitativas se describió con media, el de variables cualitativas con frecuencias. Análisis inferencial realizado con ANOVA y prueba post-hoc de Bonferroni. Se consideró estadísticamente significativo p menor a 0,05 . Resultados: $58,4 \%$ fueron de sexo masculino, la edad más frecuente fue de 29 días a 11 meses 29 días (70\%), el período con mayor número de hospitalizaciones fue entre diciembre y mayo. El tiempo promedio de oxigenoterapia fue 1.03 días, la estancia hospitalaria 1.37 días, el principal agente etiológico fue el virus sincitial respiratorio (VSR) mediante inmunocromatografía (63,8\%) y PCR (51\%). EI VSR prolonga la estancia hospitalaria y oxigenoterapia ( $p=0,001$ y $p=0,031)$. Los esquemas de terapia respiratoria: a) solución hipertónica, b) salbutamol más bromuro de ipratropio y c) salbutamol alternado con bromuro de ipratropio más salbutamol, se asociaron con menor estancia hospitalaria ( 1 a 3 días) ( $p=0.016)$. 28.8\% de pacientes recibieron antibioticoterapia, evidenciándose prolongación del tiempo de estancia hospitalaria $(p=0.000)$; los corticoides prolongaron la oxigenoterapia $(p=0,004)$. Conclusión: emplear los tres esquemas descritos previamente, disminuye la estancia hospitalaria.
\end{abstract}

Palabras claves: Bronquiolitis, terapia respiratoria, oxigenoterapia, hospitalización.

\section{ABSTRACT}

Introduction: Bronchiolitis is one of the main causes of morbidity in children under 24 months. Objective: Determine the effectiveness of respiratory therapy schemes regarding the length of hospital stay and oxygen requirement in children under 2 years old, admitted with bronchiolitis at the Metropolitan Hospital from January 2014 to December 2019. Method: Retrospective analytical study, 546 patients with bronchiolitis and oxygen therapy. Univariate analysis of quantitative variables was described with mean and qualitative variables with frequencies. ANOVA and post-hoc Bonferroni test were used for the inferential study. P value less than 0.05 was considered statistically significant. Results: $58.4 \%$ were male, the most frequent age was from 29 days to 11 months, 29 days $(70 \%)$, and the period with highest number of hospitalizations was between December and May. The average oxygen therapy time was 1.03 days, hospital stay was 1.37 days, the main etiologic agent was respiratory syncytial virus (RSV) by immunochromatography (63.8\%) and PCR (51\%). The RSV prolongs the hospital stay and oxygen therapy $(p=0.001$ and $p=0.031)$. Respiratory therapy schemes: a) hypertonic solution, $b)$ salbutamol plus ipratropium bromide and c) salbutamol alternated with ipratropium bromide plus salbutamol, were associated with a shorter hospital stay (1 to 3 days) ( $p=0.016)$. 28.8\% patients received antibiotics, which was related with a longer hospital stay $(p=0.000)$. While corticosteroids prolonged oxygen therapy ( $p=0.004)$. Conclusions: The use of the mentioned respiratory therapies, reduces the hospital stay.

Keywords: Bronchiolitis, respiratory therapy, oxygen inhalation therapy, Hospitalization.

\section{IDs Orcid}

Dayana Aylem Navarro Armas:

https://orcid.org/0000-0001-8601-9965

Daniela Alejandra Briceño Almeida: https://orcid.org/0000-0001-7341-8107 


\section{INTRODUCCIÓN}

La bronquiolitis es frecuente en menores de 2 años y genera la necesidad de hospitalización en estos pacientes; la cual genera altos costos para los padres y el sistema de salud ${ }^{1}$.

Al ser una de las patologías respiratorias con mayor afectación a una población de riesgo, es necesario determinar y generar un protocolo de manejo eficaz.

\subsection{Bronquiolitis}

Es una patología aguda que afecta al tracto respiratorio inferior, siendo el primer episodio de sibilancias, precedido por un cuadro respiratorio de rinorrea, tos, taquipnea, con o sin fiebre, que afecta a niños menores de 2 años (MJ, 2010). No todo niño con sibilancias presenta bronquiolitis ${ }^{2}$. Las manifestaciones clínicas varían según la edad del paciente, comorbilidades, exposición ambiental e historia de infecciones previas ${ }^{3}$.

El principal agente etiológico es el virus sincitial respiratorio (VSR) (80\% de los casos), virus ARN, formado por 10 genes que codifican 11 proteínas, siendo las de mayor virulencia: la proteína de fusión F, y una glicoproteína de adherencia G (Giovanni, 2014). La transmisión ocurre por inoculación de la mucosa nasofaríngea o conjuntival a través de secreciones respiratorias de personas infectadas con VSR. El periodo de incubación es de 2 a 8 días. Al afectar a la mucosa nasofaríngea, por transmisión intercelular viaja a los bronquiolos terminales, donde su replicación es más eficiente ${ }^{4}$. El infiltrado de linfocitos en los tejidos peri bronquiales y el aumento de la permeabilidad vascular, aumenta el edema de la submucosa. La pérdida de epitelio ciliado aumenta la viscosidad y la cantidad de secreciones mucosas. Todos estos eventos dan lugar a la obstrucción y atrapamiento aéreo ${ }^{3}$.

\subsection{Diagnóstico:}

Es básicamente clínico. Los primeros días se presentan síntomas respiratorios altos; posteriormente el cuadro se exacerba con tos y aumento del esfuerzo respiratorio. Para evaluar este último disponemos de la escala de Wood Downes Ferres modificada. Según su puntaje hablamos de dificultad respiratoria leve 1-3 puntos, moderada 4-7 puntos y severa 8-14 puntos. Es importante además valorar el grado de hipoxemia, con el uso de la saturación de oxígeno. La Academia americana de Pediatría (AAP) y la Organización mundial de la salud (OMS) recomiendan una saturación mayor o igual a 90\%. (Steve Cunningham, 2015) ${ }^{5}$.

No es necesaria la realización de exámenes complementarios de laboratorio, ni de imagen a menos que el paciente presente riesgo inminente de sob- reinfección bacteriana. El análisis del agente causal a través de reacción de cadena de la polimerasa no está indicado en todos los pacientes, sin embargo, el identificar al agente etiológico de la patología permite conocer el pronóstico ${ }^{6}$. En el Hospital Metropolitano se dispone de estudio por inmunocromatografía y por PCR en tiempo real.

\subsection{Tratamiento}

No todos los pacientes deben ser hospitalizados, pues la mayoría desarrollan una infección moderada auto limitada 7 . La mayoría sólo necesita medidas de soporte tales como: hidratación, antitérmicos, y oxigenoterapia con oxígeno caliente y humidificado para evitar estados de hipoxemia y lavados nasales periódicos ${ }^{8}$. El uso de broncodilatadores no ha demostrado un beneficio consistente. Flores-González JC, en el 2015 demostró mediante su estudio que combinar epinefrina con solución salina hipertónica disminuye los días de hospitalización $n^{9,3}$. Badgett (2015), et al, en su meta análisis afirma que la solución salina hipertónica redujo significativamente la duración de la estadía entre los lactantes hospitalizados ${ }^{10}$.

\section{MATERIALES Y MÉTODOS}

\subsection{Tipo de estudio}

Estudio analítico retrospectivo.

\subsection{Universo de estudio y muestra}

546 pacientes menores de 2 años ingresados en el Hospital Metropolitano, en el periodo comprendido entre el 01 de enero del 2014 al 31 de diciembre del 2019 con diagnóstico de bronquiolitis. El método de muestreo empleado fue no probabilístico. La determinación del tamaño de la muestra, se realizó por medio de la fórmula para cálculo de una población infinita con una prevalencia de 35\% siendo analizados en total 546 pacientes.

Se realizaron los siguientes criterios de inclusión:

- Niños menores de 2 años con diagnóstico de bronquiolitis.

- Niños con necesidad de apoyo con oxígeno con ese diagnóstico en ese periodo.

Los criterios de exclusión para el estudio fueron:

- Niños/as menores de dos años con signos sugestivos de asma y/o que se encuentren premedicados, mediante la utilización de broncodilatadores, dos semanas previas.

- Niños/as que hayan sido hospitalizados o diagnosticados previamente con eventos sibilantes recurrentes. 
- Niños/as menores de 2 años con diagnóstico de bronquiolitis que hayan sido hospitalizados sin requerimiento de oxígeno.

- Niños/as menores de dos años alérgicos, que hayan usado antihistamínicos, inhibidores de los leucotrienos, corticoides 2 semanas previas al ingreso.

- Niños/as menores de 2 años inmunodeprimidos.

- Niños/as con bronquiolitis que hayan ingresado directamente a terapia intensiva pediátrica o neonatal y que hayan ameritado ventilación mecánica.

- Niños/as con enfermedades crónicas pulmonares: fibrosis quística y displasia broncopulmonar.

- Niños mayores de 24 meses

\subsection{Técnica de análisis}

La base de datos y el análisis se realizó mediante el paquete estadístico SPSS (por sus siglas en inglés: Stadistical Package for the Social Sciences). El análisis univariado de variables cualitativas fue descrito con frecuencias absolutas y relativas.

El análisis univariado de variables cuantitativas fue descrito con medidas de tendencia central (media) y medidas de dispersión (error estándar de la media). El análisis univariado de variables cualitativas fue descrito con frecuencias absolutas y relativas. Para el estudio inferencial se utilizó el ANOVA (por sus siglas en inglés: ANalysis Of VAriance) con la finalidad de determinar diferencias significativas entre los grupos de estudio y la prueba post-hoc de Bonferroni. La asociación se realizó con razón de momios de prevalencia e intervalo de confianza (IC) 95\%. Para la comparación entre la respuesta de cada terapia respiratoria instaurada se utilizó diferencia de medias, puntaje.

\section{RESULTADOS}

\subsection{Estadística descriptiva}

\subsubsection{Caracterización de la población}

La población estuvo constituida por 546 pacientes menores de 24 meses diagnosticados de bronquiolitis en el Hospital Metropolitano de Quito, durante el periodo de enero del 2014 a diciembre del 2019.

\subsubsection{Distribución de la población acorde al mes de ingreso}

Se observó que durante los 5 años los meses más frecuentes en los que fueron ingresados pacientes menores de dos años con bronquiolitis fueron enero con $21,79 \%$ (119), seguido de febrero $20,70 \%$ (113), marzo $11.72 \%$ (64), abril 9,16\% (50), diciem- bre $8.61 \%$ (47), en el resto de meses se evidencia un decremento paulatino de la cantidad de ingresos hospitalarios.

\subsubsection{Distribución de la población acorde a la edad}

El rango de edad más frecuente fue de 29 días a 11 meses 29 días representando el $70 \%$, seguido por pacientes entre los 12 a 23 meses con 29 días que constituyen el $25.4 \%$ y finalmente el $4.6 \%$ conformado por los menores de 29 días. La media fue 21.84 meses, con una desviación estándar de 13.24 meses y con un rango de 29 días a 11 meses 29 días.

\subsubsection{Distribución del sexo de la población}

De los 546 pacientes menores de 24 meses el $58.42 \%$ (319) fueron hombres y $41.58 \%$ (227) fueron mujeres.

\subsubsection{Severidad de dificultad respiratoria al ingreso} según la escala de Wood Downes Ferres modificada

De los 546 pacientes de nuestro estudio 89.93\% (491) presentaron al ingreso dificultad respiratoria leve y $10.07 \%$ (55) presentaron dificultad respiratoria moderada.

\subsubsection{Etiología}

De los 546 pacientes incluidos, se realizó panel respiratorio a $78.9 \%$ pacientes (431), de los cuales $60,3 \%$ (329) fueron por inmunocromatografía y $18.7 \%$ (102) por PCR en tiempo real. Por inmunocromatografía se obtuvieron los siguientes resultados: $63,8 \%$ (210) fueron positivos para VSR, 0.9\% (3) para influenza A, y $0,6 \%$ (2) para adenovirus. Mientras que 34,7\% (114) fueron negativos. Por PCR en tiempo real se observó predominio de VSR 51\% (52) de los casos, seguido por otros agentes virales: parainfluenza 18,6\% (19), rinovirus, 8,8\% (9), metaneumovirus $7.8 \%$ (8), VSR y rinovirus en 4,9\% (5), influenza y adenovirus en un 2,9\% (3), más de dos microrganismos $1 \%$ (1) y reporte negativo $1 \%$ (1).

\subsubsection{Esquemas de terapia respiratoria}

Se identificaron 13 diferentes esquemas de terapia respiratoria, cuya frecuencia de utilización se demuestra en la Tabla 1. 
Tabla 1. Esquemas de terapia respiratoria

\begin{tabular}{lc} 
Salbutamol & $21.98 \%$ \\
\hline Solución hipertónica + Salbutamol & $13.37 \%$ \\
\hline Salbutamol + Bromuro de ipratropio & $10.6 \%$ \\
\hline $\begin{array}{l}\text { Solución hipertónica alternada con Adrenalina } \\
\text { racémica }\end{array}$ & $8.4 \%$ \\
\hline Salbutamol más Ambroxol & $8.1 \%$ \\
\hline $\begin{array}{l}\text { Salbutamol alternado con Adrenalina } \\
\text { racémica }\end{array}$ & $7.7 \%$ \\
\hline $\begin{array}{l}\text { Salbutamol alternado con Solución } \\
\text { hipertónica }\end{array}$ & $5.9 \%$ \\
\hline $\begin{array}{l}\text { Bromuro de ipratropio+ Salbutamol alternado } \\
\text { con Solución hipertónica }\end{array}$ & $5.5 \%$ \\
\hline $\begin{array}{l}\text { Adrenalina racémica alternada con Salbuta- } \\
\text { mol + Bromuro de ipratropio }\end{array}$ & $4.8 \%$ \\
\hline \begin{tabular}{l} 
Solución hipertónica \\
\hline $\begin{array}{l}\text { Adrenalina racémica + Dexametasona alter- } \\
\text { nada con Adrenalina racémica + Ambroxol }\end{array}$
\end{tabular} & $4.2 \%$ \\
\hline \begin{tabular}{l} 
Adrenalina racémica \\
\hline $\begin{array}{l}\text { Salbutamol alternada con Salbutamol + Bro- } \\
\text { muro de ipratropio }\end{array}$
\end{tabular} & $2.6 \%$ \\
\hline
\end{tabular}

\subsubsection{Sistema de oxígeno de flujo bajo}

De la población estudiada 99,63\% (544) utilizaron oxígeno por cánula nasal y $0.37 \%$ (2) utilizaron mascarilla de oxígeno simple.

\subsubsection{Tiempo de requerimiento de oxígeno}

Al categorizar el tiempo de requerimiento de oxígeno se evidenció que el 49.3\% (269) de pacientes utilizaron oxígeno entre 1 a 3 días, 24.5\% (134) menos de 1 día, 24\% (131) más de 3 hasta 7 días y 2.2\% (12) más de 7 días. La media fue de 1.04 días, con una desviación estándar de 0.757 .

\subsubsection{Tiempo de estancia hospitalaria}

En el presente trabajo se observó que el tiempo de estancia hospitalaria fue de 1 a 3 días en el 60,6\% (331), seguido de un periodo de más de 3 hasta 7 días en $33.7 \%$ (184), más de 7 días en 3.1\% (17) y finalmente < de 1 día en 2.6\% (14). La media fue de 1.37 días, con desviación estándar de 0.590 .

\subsubsection{Hallazgos de la radiografía de tórax}

Se realizó radiografía de tórax a un 96.5\% (527) de los pacientes estudiados, siendo el hallazgo más frecuente la hiperinsuflación 51.1\% (279), seguido por engrosamiento peribronquial $31.5 \%$ (172), infiltrado $13.2 \%(72)$, atelectasia $0.5 \%$ (3) y finalmente consolidado $0.2 \%(1)$.

\subsubsection{Antibioticoterapia:}

Se administró antibiótico al 28.8\% (158) de pacientes y el $71.2 \%$ (389) no recibieron antibióticos. Los antibióticos empleados fueron: cefuroxima $32.1 \%$
(50), ceftriaxona 25\% (39), claritromicina 14.1\% (22), amoxicilina 9\% (14), amoxicilina más ácido clavulánico 7.7\% (12), azitromicina 5.85\% (9), otros 5.8\% (9) y ampicilina sulbactam $0.6 \%$ (1). La vía de administración de antibiótico más frecuente fue intravenosa en $65.2 \%$ (103), la vía oral representó el 34.8\% (55). El 51.9\% (82), recibieron antibiótico por un periodo de 1 a 3 días, $41.1 \%$ (65) más de 3 días hasta 7 días, $3,8 \%(6)<$ de 1 día y $3.2 \%$ (5) más de 7 días; con una media de 1.44 días y desviación estándar de 0.623 días.

Los motivos de administración de antibioticoterapia fueron: radiografía de tórax con infiltrado o consolidado 24.8\% (39), no justificado 22.9\% (36), exámenes de laboratorio con datos de sobreinfección bacteriana $21.7 \%$ (34), otitis $16.6 \%$ (26), inmunomodulador 10.2\% (16), sinusitis 3.2\% (5) e infección de vías urinarias $0.6 \%(1)$.

\subsubsection{Administración de corticoides}

Se administró corticoide al 40.1\% (219) de pacientes, mientras que $59.5 \%$ (327) no recibieron corticoide. Se identificaron 7 tipos de corticoide dentro del estudio, siendo el más frecuente la metilprednisolona con $34.4 \%$ (75), seguida de la prednisolona $24.3 \%$ (53), fluticasona 19.3\% (42), prednisona 9.6\% (21), dexametasona 5\% (11), mometasona 4.1\% (9) y finalmente budesonida 3.2\% (7). Se registró una vía de administración intravenosa de corticoide en el $37.6 \%$ (82), oral $35.3 \%$ (77) e inhalado $27.1 \%$ (59). El rango de administración de corticoide fue de 1 a 3 días en el 63.8\% (139), entre 3 y 7 días 30.3\% (66), menos de 1 día $4,1 \%$ (9) y más de 7 días $1.8 \%$ (4). La media fue de 1.29 días con desviación estándar de 0.574 .

\subsection{Estadística inferencial}

3.2.1 Esquema de terapia respiratoria con respecto al tiempo de requerimiento de oxígeno y la estancia hospitalaria

Se estableció diferencias significativas $(p=0,016)$ entre el tipo de terapia respiratoria y la estancia media (Figura 1), no así con el tiempo de requerimiento de oxígeno $(p=0,388)$. Se determinó que los esquemas de terapia respiratoria: a) solución hipertónica, b) salbutamol más bromuro de ipratropio y, c) salbutamol alternado con bromuro de ipratropio más sabutamol se relacionan con menor estancia hospitalaria (media $=1$ a 3 días) que el resto de terapias $(p=0.016)$. 


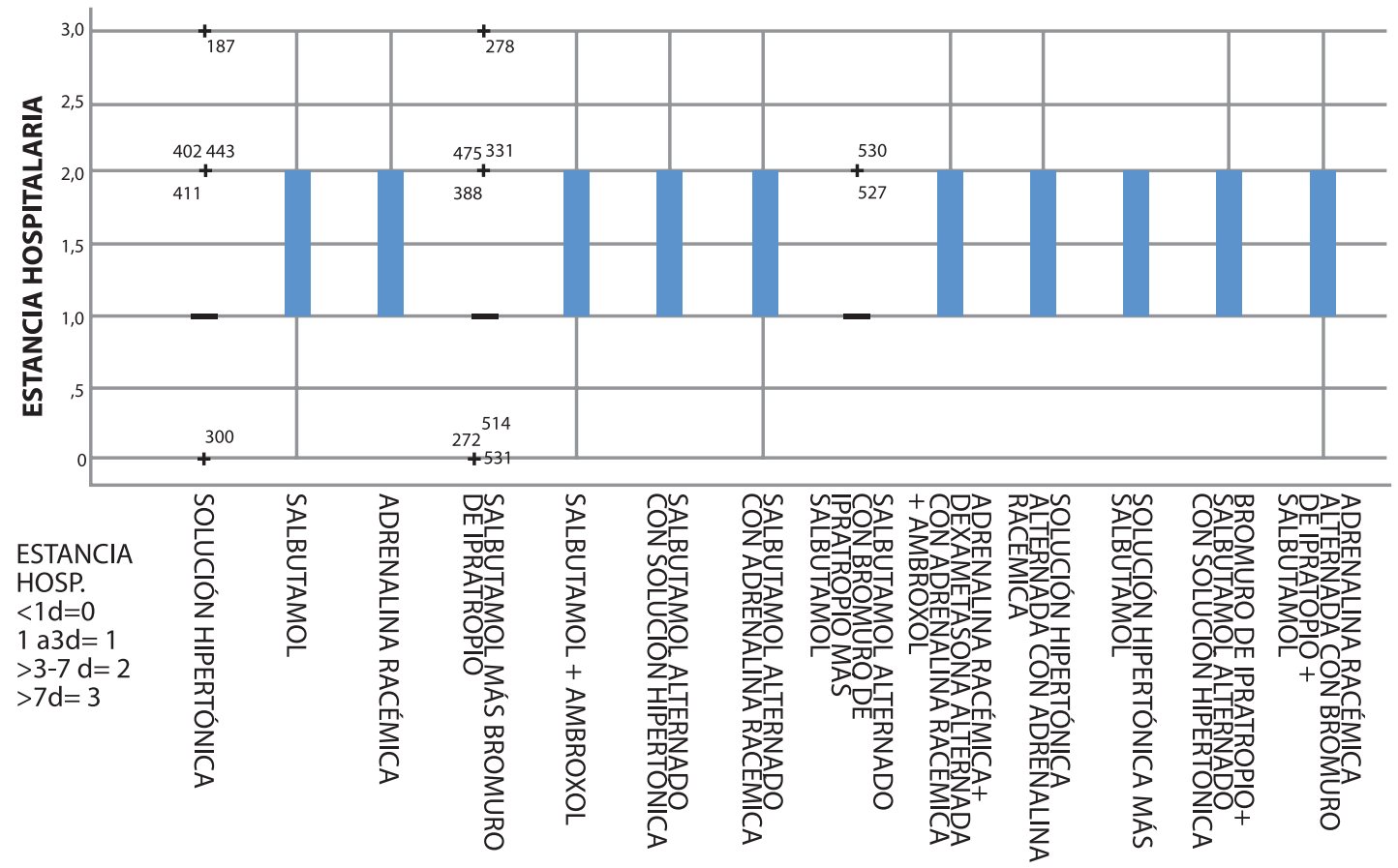

Figura 1. Esquema de terapia respiratoria con respecto al tiempo de estancia hospitalaria

Fuente: Los autores

\subsubsection{Correlación de la edad con el tiempo de requerimiento de oxígeno y de estancia hospitalaria}

Se estableció que existen diferencias estadísticamente significativas al correlacionar la edad con el tiempo de requerimiento de oxígeno y la estancia hospitalaria $p=0.000$ y $p=0.008$ respectivamente. Los pacientes menores de 29 días permanecen más tiempo hospitalizados y con requerimiento de oxígeno (media = más de 7 días). (Figura 2)

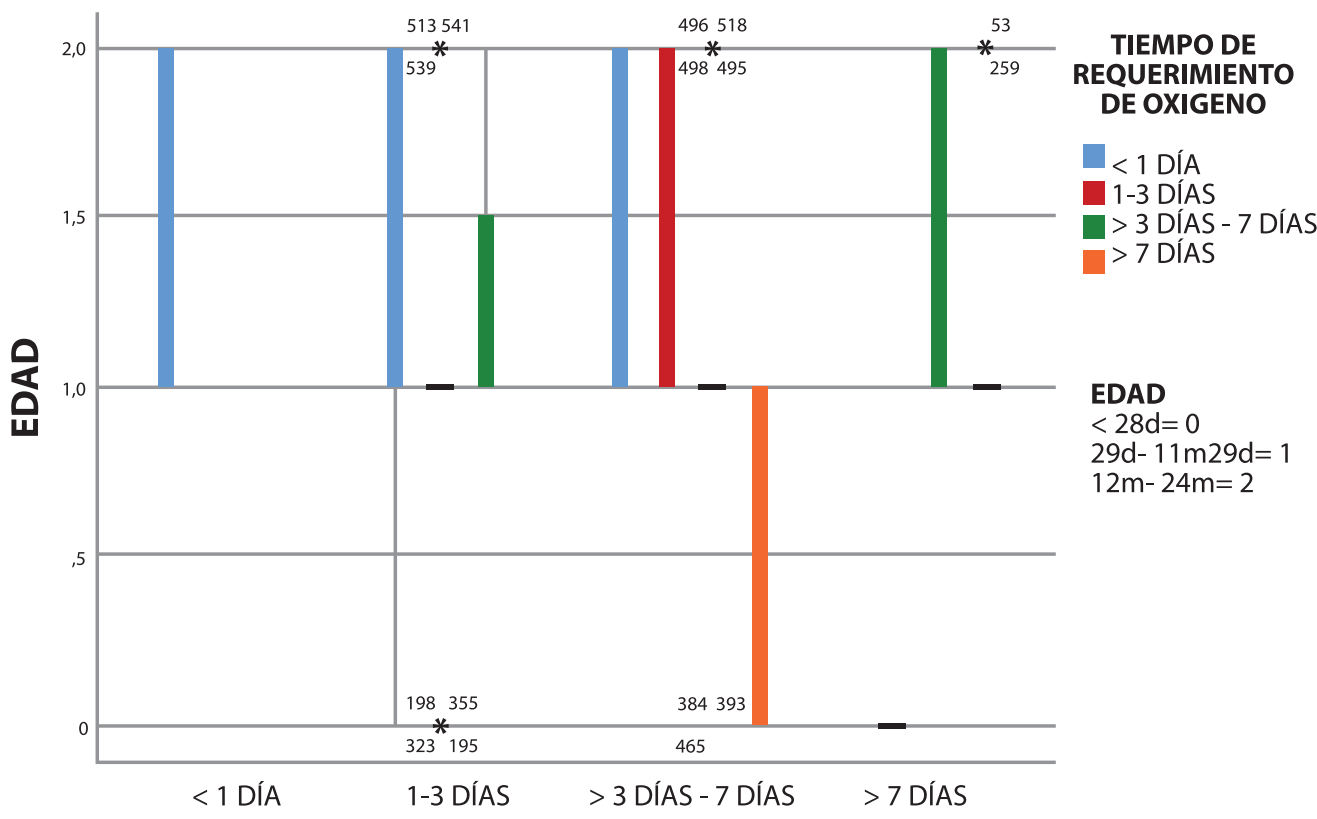

ESTANCIA HOSPITALARIA

Figura 2. Correlación de la edad con el tiempo de requerimiento de oxígeno y de estancia hospitalaria

Fuente: Los autores 


\subsubsection{Asociación del tipo de agente causal con el tiempo de estancia hospitalaria y de oxigenoterapia}

Se observaron diferencias significativas entre el agente causal y el tiempo de oxígeno ( $p=0.000$ ), así como con la estancia hospitalaria $(p=0.031)$. Se determinó que el VSR se relacionó con mayor tiempo de estancia hospitalaria y de requerimiento de oxígeno en ambos paneles respiratorios (media = más de 7 días). (Figura 3)

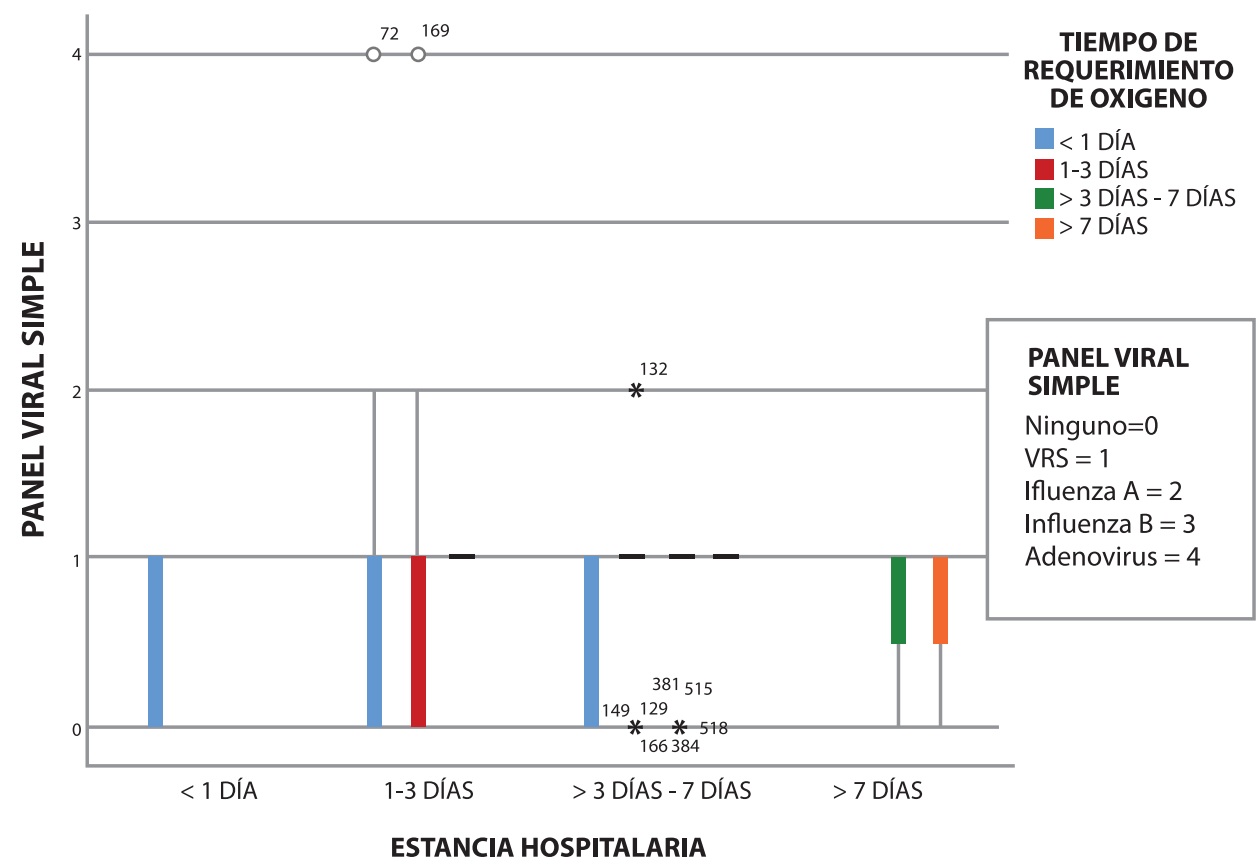

Figura 3. Correlación del tipo de agente causal detectado por panel viral simple con el tiempo de estancia hospitalaria y de oxigenoterapia

Fuente: Los autores

3.2.4 Comparación de la utilización de antibioticoterapia en relación al tiempo de la estancia hospitalaria y de oxigenoterapia

Existe relación estadísticamente significativa entre la administración de antibiótico con respecto al tiempo de estancia hospitalaria $(p=0.000)$, mas no con el tiempo de requerimiento de oxígeno $(p=0.62)$. Los pacientes que no recibieron antibioticoterapia permanecieron hospitalizados menos tiempo. (Figura 4).

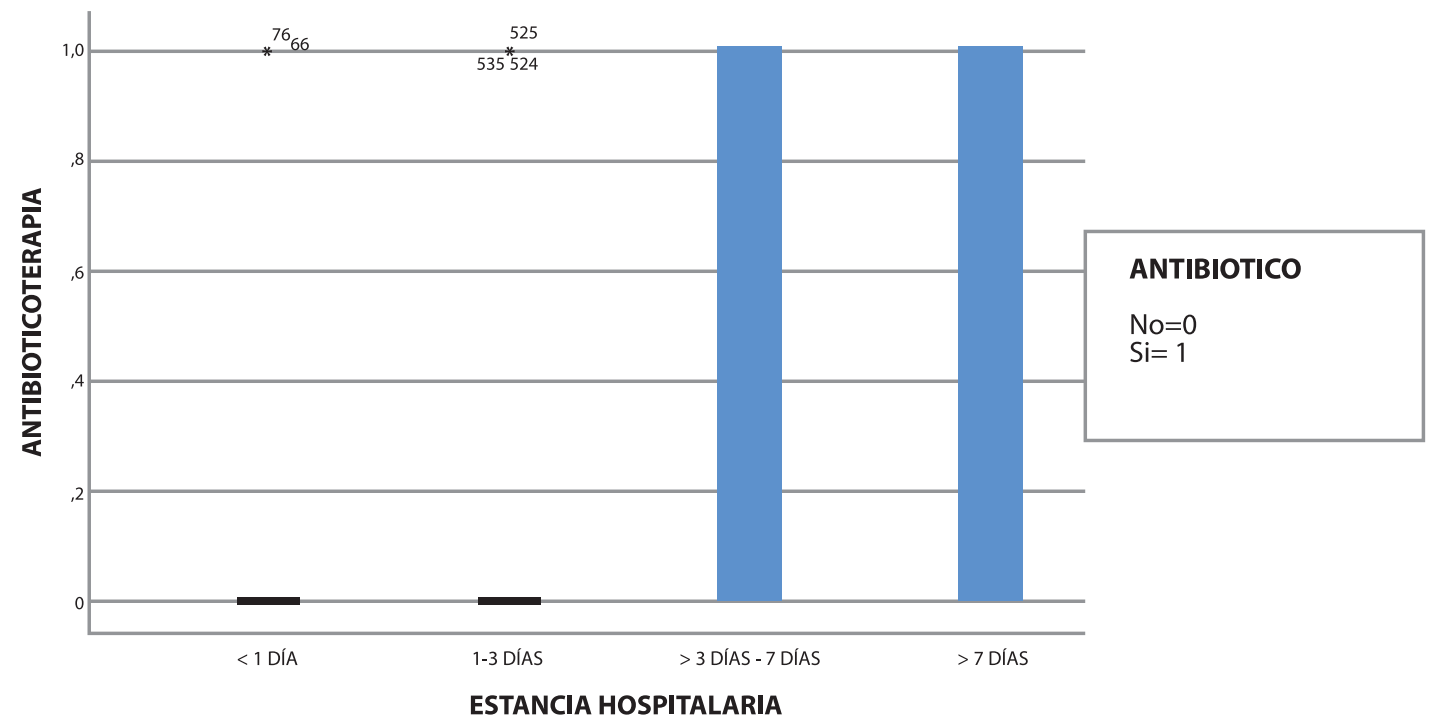

Figura 4. Relación de la administración de antibiótico con el tiempo de estancia hospitalaria

Fuente: Los autores 


\subsubsection{Comparación de la utilización de corticoides en relación al tiempo de la estancia hospitalaria y de oxi- genoterapia}

Existe relación estadísticamente significativa entre el uso de corticoide con respecto al tiempo de requerimiento de oxígeno p=0.004 (Figura 5), mas no con la estancia hospitalaria p=0.051.

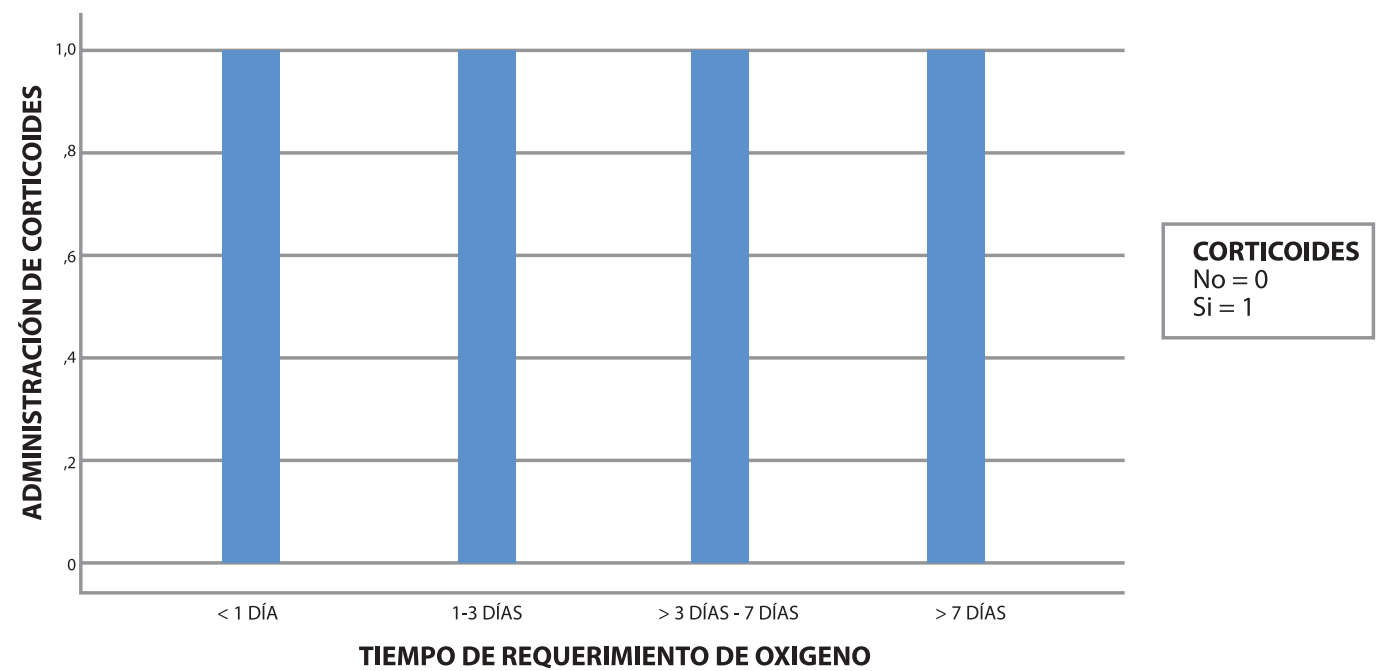

Figura 5. Comparación entre la utilización de corticoide con el tiempo de requerimiento de oxígeno

Fuente: Los autores

\section{DIscusıón}

En nuestro estudio se observó que el período en donde existió mayor frecuencia de hospitalizaciones fue entre diciembre a mayo, lo cual concordó con lo registrado por Rivera-Sepúlveda; y que se explica porque en temperaturas bajas, como las evidenciadas en estos meses, los cilios de las vías respiratorias disminuyen su motilidad, permitiendo fácilmente el paso de microorganismos hacia los bronquiolos y el desarrollo de infecciones ${ }^{11}$. En el presente trabajo se analizaron 546 casos de bronquiolitis en pacientes menores de 2 años en el servicio de Pediatría de un hospital privado de la ciudad de Quito en un período de 6 años. De los cuales, el 58,4\% fueron de sexo masculino y el $41,5 \%$ de sexo femenino; hallazgos similares a los observados por Parra y colaboradores (2013), quienes identificaron que la hospitalización por bronquiolitis se produjo con más frecuencia en varones que en niñas (62 vs 38\%), con un predominio en los menores de 12 meses de edad $^{12}$, lo que también coincidió con la distribución de la población estudiada en la que se identificó que la edad más frecuente para la presentación de la enfermedad fue de 29 días a 11 meses 29 días de vida (70\%), seguidos de los niños entre 12 meses a 23 meses 29 días $(25,4 \%)$ y finalmente por los menores de 29 días (4,6\%). Hecho que se deduce en base a las características anatomofuncionales del árbol bronquial en los menores de 2 años, en quienes los alveolos no se encuentran completamente desarrollados y además carecen de los poros de Kohn y los canales de Lambert ${ }^{13}$.
Estudios epidemiológicos a nivel internacional identificaron al virus sincitial respiratorio como la principal causa de bronquiolitis en un 70 a $80 \%$ de casos, con un predominio del $75 \%$ en el primer año de vida ${ }^{14}$. Lo cual se relacionó con nuestros hallazgos en donde se emplearon métodos de laboratorio para la identificación del agente etiológico en un 78.9\%, mediante técnicas de inmunocromatografía en el $60,3 \%$ y PCR en tiempo real en un 18,7\%; encontrándose en ambos casos al virus sincitial respiratorio como el principal agente implicado (63,8 y 51\% respectivamente), seguido de otros agentes virales en menor frecuencia, tales como parainfluenza, rinovirus, metaneumovirus, entre otros. En el estudio de Sruamsiri $R$ (2018), se demostró que en 6811 pacientes menores de 5 años diagnosticados de virus sincitial respiratorio, el tiempo de estancia hospitalaria se prolongó hasta 7.5 días $(p=0.01)^{15}$, lo que concordó con nuestro grupo de pacientes estudiados en los cuales se observó que la presencia de infección por virus sincitial respiratorio se asoció con mayor tiempo de hospitalización así como de requerimiento de oxígeno. $(p=0.000), y(p=0.031)$ respectivamente.

El promedio de tiempo de duración de oxigenoterapia en poblaciones del primer mundo es de 24 horas $^{16,17}$. Al analizar los datos obtenidos en este trabajo se observó que la media de tiempo de oxigenoterapia fue de 1.03 días, lo cual coincidió con los datos descritos en otros escenarios.

En cuanto a la terapia respiratoria que se empleó en la población estudiada se identificaron 13 esque- 
mas, de los cuales los más frecuentes fueron: a) salbutamol b) solución hipertónica más salbutamol, c) salbutamol más bromuro de ipratropio, d) solución hipertónica alternada con adrenalina racémica y e) múltiples combinaciones.

La solución salina hipertónica al 3\% nebulizada ha sido estudiada desde hace más de 10 años, y de acuerdo a la última revisión de la biblioteca Cochrane sobre el tema, tras realizar un análisis de 28 estudios que incluyeron a 4195 lactantes, la misma se relacionó con una disminución de la estancia hospitalaria en 10 horas al compararse con solución salina simple al 0,9\% ( $p=0.001)$, además se identificó mejoría en las puntuaciones de dificultad respiratoria en los niños hospitalizados y disminución del riesgo de hospitalización en 14\% de los pacientes manejados ambulatoriamente o en emergencia con este tipo de terapia ${ }^{18}$. Por lo que la misma puede emplearse como un método seguro y de amplia disponibilidad en el tratamiento de la bronquiolitis. Este dato se asoció con nuestro trabajo en donde se constató que los pacientes que recibieron terapia respiratoria con solución hipertónica, salbutamol más bromuro de ipratropio y salbutamol alternado con bromuro de ipratropio más salbutamol presentaron menor estancia hospitalaria (rango $=1$ a 3 días) que el resto de terapias $(p=0.016)$. En cuanto al empleo de adrenalina racémica, Teerani en Canadá en el año 2016 realizó un análisis a 102 pacientes hospitalizados con bronquiolitis, comparando adrenalina racémica, salbutamol y solución salina al 0,9\%, y determnó que ninguna de las soluciones se relacionó con modificaciones en la estancia hospitalaria ${ }^{19}$, lo cual concuerda con nuestro estudio.

Investigaciones realizadas en Reino Unido indicaron que el promedio de estancia fue de 66 horas $^{17}$. Datos que fueron similares a los de nuestra investigación, en donde la media de estancia fue de 1,37 días, con rango de 1 a 3 días en el $60.6 \%$ de los casos y más de 7 días en 3,1\%.

La radiografía de tórax no está recomendada de forma rutinaria en pacientes con bronquiolitis no complicada ${ }^{15}$, se realizaron varios estudios intentando implementar una práctica de atención con calidad, según Wrotek, A y colaboradores (2019), en su estudio determinaron que los niños en los que se realizó radiografía de tórax independientemente del resultado tuvieron un riesgo 22.9 veces mayor de tratamiento con antibióticos y requirieron una estadía hospitalaria más prolongada que aquellos sin ésta (10 frente a 8 días, respectivamente; $p<0.01)^{20}$. Sin embargo en nuestro estudio se evidenció que en el $96.5 \%$ de los pacientes estudiados se realizó radiografía de tórax, teniendo en cuenta que sólo el $10.07 \%$ presentaron a su ingreso dificultad respiratoria moderada, por lo tanto este estudio se efectuó innecesariamente.
Los antibióticos no han formado parte de las recomendaciones terapéuticas internacionales, así lo corroboraron las guías de la AAP y NICE, quienes han aconsejado utilizar los mismos sólo en caso de infección bacteriana asociada o mientras haya una fuerte sospecha de la misma ${ }^{16,17}$.

Según un estudio prospectivo realizado por Gomaa MA y colaboradores (2012), en el que se incluyó a 180 pacientes con bronquiolitis, de los cuales el $55.6 \%$ tuvo otitis media aguda, siendo más común la unilateral con $65 \%$, en quienes se realizaron frotis con Gram más cultivo y ensayos inmunoabsorbentes ligados a enzimas (ELISA) en aspirados del oído medio, se encontró en $86 \%$ patógenos bacterianos y en $14 \%$ VRS respectivamente, por lo tanto se justifica su uso $^{21}$.

Existen varios estudios sobre el uso de antibióticos con efecto inmunomodulador en bronquiolitis, en los que no se evidenció reducción significativa del virus a las 48 horas después del tratamiento con azitromicina según Zhang. $Y$, et al. (2019) ; además tampoco se constató que acorte la estancia hospitalaria $P=0,08$ o el tiempo de suministro de oxígeno $p=0.10,{ }^{22}$ según una revisión sistemática y meta análisis realizado por Si-Yi, C, et al (2019), se demostró que acortó el tiempo para el alivio de sibilancias $p=$ 0.007 y tos $p<0.00001$ y también reveló que la terapia con azitromicina redujo significativamente las tasas de detección de Streptococcus pneumoniae $(p=0.0006)$, Haemophilus $(p=0.0002)$, y Moraxella catarrhalis $(p<0.00001)$ en la región nasofaríngea ${ }^{23}$.

Al analizar la relación de la administración de antibiótico con el tiempo de estancia hospitalaria y requerimiento de oxígeno se demostró que existe relación estadísticamente significativa con el tiempo de estancia hospitalaria. $\mathrm{p}=0.000$, más no con el tiempo de requerimiento de oxígeno $\mathrm{p}=0.62$. Determinando que aquellos pacientes que no recibieron antibioticoterapia permanecieron hospitalizado menos tiempo.

Estudios realizados en España por Alarcón Andrade y Cifuentes, incluyeron cuatro revisiones sistemáticas con 20 estudios aleatorizados en los que concluyeron que el uso de corticoides sistémicos no aportó ningún beneficio en el tratamiento de la bronquiolitis, ni siquiera en pacientes con ventilación mecánica, debido a que aumentó las cargas virales de VRS en la vía aérea ${ }^{24}$; hecho que coincidió con nuestro análisis en el pudimos evidenciar que si bien existió correlación entre la administración de corticoides y el tiempo de requerimiento de oxígeno no sucedió lo mismo con el tiempo de estancia hospitalaria.

\section{CONCLUSIONES}

Los esquemas de terapia respiratoria que demostraron tener una relación significativa respecto a menor 
tiempo de estancia hospitalaria fueron: a) solución hipertónica, b) salbutamol más bromuro de ipratropio y c) salbutamol alternado con bromuro de ipratropio más salbutamol. No así con relación al tiempo de requerimiento de oxígeno.

- La edad repercutió sobre el tiempo de estancia hospitalaria en pacientes menores de 29 días, los cuales permanecieron mayor tiempo hospitalizados y con oxígeno.

- El sexo de los pacientes no se asocia con el tiempo de estancia hospitalaria ni de requerimiento de oxígeno.

- El virus respiratorio sincitial influye prolongando el tiempo de estancia hospitalaria y de oxigenoterapia.

- Los pacientes que no recibieron antibioticoterapia permanecieron hospitalizados por menor tiempo.

- Existe asociación significativa entre el uso de corticoides y el tiempo de requerimiento de oxígeno, no así con la estancia hospitalaria.

\section{CONTRIBUCIÓN DE LOS AUTORES}

Dayana Navarro: Concepción y diseño del trabajo; recolección y obtención de resultados; análisis e interpretación de datos; redacción del manuscrito.

Daniela Briceño: Concepción y diseño del trabajo; recolección y obtención de resultados; análisis e interpretación de datos; redacción del manuscrito.

\section{CONFLICTO DE INTERESES}

Los autores declararon no tener ningún conflicto de interés personal, financiero, intelectual, económico y de interés corporativo con el Hospital Metropolitano y los miembros de la revista MetroCiencia.

\section{REFERENCIAS BIBLIOGRÁFICAS}

1. Ros Pérez P, Otheo de Tejada E, Pérez Rodríguez MJ. Bronquiolitis en pediatría. Inf Ter del Sist Nac Salud [Internet]. 2010;34(1):311. Available from: http://dialnet.unirioja.es/servlet/articulo?codigo=3646804\&info=resumen\&idioma $=E N G$

2. Mukherjee S, Rutter K, Watson L, Eisenhut M. Adverse effects of bronchodilators in infants with bronchiolitis [Internet]. Vol. 20, Journal of Pediatric Pharmacology and Therapeutics. Pediatric Pharmacy Advocacy Group, Inc.; 2015. p. 70-1. Available from: https://www.ncbi. nlm.nih.gov/pmc/articles/PMC4353204/

3. Flores $\mathbf{P}$, Mendes $\mathbf{A L}$, Neto AS. A randomized trial of nebulized $3 \%$ hypertonic saline with salbutamol in the treatment of acute bronchiolitis in hospitalized infants. Pediatr Pulmonol [Internet]. 2016 Apr 1;51(4):418-25. Available from: https://pubmed.ncbi.nlm.nih. gov/26334188/

4. Martinello RA, Chen MD, Weibel C, Kahn JS. Correlation between respiratory syncytial virus genotype and severity of illness. J Infect Dis [Internet]. 2002 Sep 15;186(6):839-42. Available from: https:// pubmed.ncbi.nlm.nih.gov/12198620/

5. Cunningham S, Rodriguez A, Adams T, Boyd KA, Butcher I, Enderby $\mathbf{B}$, et al. Oxygen saturation targets in infants with bronchiolitis (BIDS): A double-blind, randomised, equivalence trial. Lancet [Inter- net]. 2015 Sep 12; 386(9998):1041-8. Available from: http://www.

6. Piedimonte G, Perez MK. Respiratory syncytial virus infection and bronchiolitis. Pediatr Rev [Internet]. 2014 Dec 1; 35(12):519-30. Available from: https://www.ncbi.nlm.nih.gov/pmc/articles/PMC5029757/

7. Mansbach JM, Clark S, Piedra PA, Macias CG, Schroeder AR, Pate BM, et al. Hospital course and discharge criteria for children hospitalized with bronchiolitis. J Hosp Med [Internet]. 2015 Apr 1;10(4):20511. Available from: /pmc/articles/PMC4390446/?report=abstract

8. Hutchings FA, Hilliard TN, Davis PJ. Heated humidified high-flow nasal cannula therapy in children [Internet]. Vol. 100, Archives of Disease in Childhood. BMJ Publishing Group; 2015. p. 571-5. Available from: https://pubmed.ncbi.nlm.nih.gov/25452315/

9. Fernandes RM, Bialy LM, Vandermeer B, Tjosvold L, Plint AC, Patel $\mathbf{H}$, et al. Glucocorticoids for acute viral bronchiolitis in infants and young children [Internet]. Vol. 2013, Cochrane Database of Systematic Reviews. John Wiley and Sons Ltd; 2013. Available from: https:// pubmed.ncbi.nlm.nih.gov/23733383/

10. Badgett RG, Vindhyal M, Stirnaman JT, Gibson CM, Halaby R. A living systematic review of nebulized hypertonic saline for acute bronchiolitis in infants [Internet]. Vol. 169, JAMA Pediatrics. American Medical Association; 2015. p. 788-9. Available from: https://pubmed. ncbi.nlm.nih.gov/26099054/

11. Christopher AB, Ochoa S, Krushansky E, Francis R, Tian X, Zahid $\mathbf{M}$, et al. The Effects of Temperature and Anesthetic Agents on Ciliary Function in Murine Respiratory Epithelia. Front Pediatr [Internet]. 2014 Oct 16; 2(OCT):111. Available from: http://journal.frontiersin.org/article/10.3389/fped.2014.00111/abstract

12. Parra A, Jiménez C, Hernández S, García JE, Cardona ÁM. Bronquiolitis: artículo de revisión. Neumol pediátr [Internet]. 2013;8(8):95101. Available from: https://pesquisa.bvsalud.org/portal/resource/pt/ lil-701696?lang=es

13. John E. Cotes, David J. Chinn, Martin R. Miller. Lung Function: Physiology, Measurement and Application in Medicine, 6th Edition | Wiley [Internet]. 1st ed. Hoboken, Nueva Jersey: Wiley-Blackwell; 2006. 648 p. Available from: https://www. wiley.com/en-us/Lung+Function\%3A+Physiology\%2C+Measurement+and+Application+in+Medicine\%2C+6th+Edition-p-9780632064939

14. Castaños C, Rodríguez S. GAP 2013: Manejo de la Bronquiolitis. Argentina: Hospital de Pediatría Garrahan; 2019. 27 p.

15. Sruamsiri R, Kubo H, Mahlich J. Hospitalization costs and length of stay of Japanese children with respiratory syncytial virus: A structural equation modeling approach. Med (United States) [Internet]. $2018 \mathrm{Jul}$ 1;97(29). Available from: /pmc/articles/PMC6086555/?report=abstract

16. Ralston SL, Lieberthal AS, Meissner HC, Alverson BK, Baley JE, Gadomski AM, et al. Clinical practice guideline: The diagnosis, management, and prevention of bronchiolitis [Internet]. Vol. 134, Pediatrics. American Academy of Pediatrics; 2014. p. e1474-502. Available from: https://pubmed.ncbi.nlm.nih.gov/25349312/

17. National Institute for Health and Care Excellence. Bronchiolitis diagnosis and management of bronchiolitis in children. Http://WwwNiceOrgUk/Guidance/Ng9 [Internet]. 2015;1-301. Available from: https://www.ncbi.nlm.nih.gov/books/NBK299243/

18. Zhang L, Mendoza-Sassi RA, Wainwright C, Klassen TP. Nebulised hypertonic saline solution for acute bronchiolitis in infants [Internet]. Vol. 2017, Cochrane Database of Systematic Reviews. John Wiley and Sons Ltd; 2017. Available from: https://pubmed.ncbi.nlm.nih. gov/29265171/

19. Sakulchit T, Goldman RD. Nébulisation d'épinéphrine chez les jeunes enfants atteints de bronchiolite. Can Fam Physician [Internet]. 2016 Dec 1;62(12):991. Available from: www.cfp.ca

20. Wrotek A, Czajkowska M, Jackowska T. Chest Radiography in Children Hospitalized with Bronchiolitis. In: Advances in Experimental Medicine and Biology [Internet]. Springer; 2019. p. 55-62. Available from: https://pubmed.ncbi.nlm.nih.gov/31529287/

21. Gomaa MA, Galal O, Mahmoud MS. Risk of acute otitis media in relation to acute bronchiolitis in children. Int J Pediatr Otorhinolaryngol [Internet]. 2012 Jan [cited 2020 Dec 16];76(1):49-51. Available from: https://pubmed.ncbi.nlm.nih.gov/22018925/

22. Zhang Y, Dai J, Jian H, Lin J. Effects of macrolides on airway microbiome and cytokine of children with bronchiolitis: A systematic review and meta-analysis of randomized controlled trials [Internet]. Vol. 63, Microbiology and Immunology. Blackwell Publishing Asia; 2019. p. 343-9. Available from: https://pubmed.ncbi.nlm.nih.gov/31283028/ 
23. Che SY, He H, Deng Y, Liu EM. Clinical effect of azithromycin adjuvant therapy in children with bronchiolitis: A systematic review and Meta analysis. Chinese J Contemp Pediatr [Internet]. 2019 Aug 25;21(8):812-9. Available from: https://pubmed.ncbi.nlm.nih. gov/31416508/
24. Alarcón-Andrade G, Cifuentes L. ¿Deben utilizarse corticoides sistémicos en la bronquiolitis? Medwave [Internet]. 2018 May 7;18(3):e7207. Available from: /link.cgi/Medwave/PuestaDia/ResEpis/7207.ac 